\title{
Instability helps virtual flies to mate
}

\author{
Thorsten Hüls* \\ Fakultät für Mathematik \\ Universität Bielefeld \\ Postfach 100131, 33501 Bielefeld \\ Germany \\ huels@mathematik.uni-bielefeld.de
}

August 26, 2004

\begin{abstract}
In this paper we perform a bifurcation analysis for a discrete time dynamical system, describing the behavior of a virtual fly, developed by Böddeker and Egelhaaf in [2]. Like real blowflies, the virtual counterparts exhibit a dichotomous behavior: they catch small targets but follow big objects in a constant distance. We consider this model for targets on linear and on circular trajectories. Then we transform the system into a "frozen" form, such that the position of the target is fixed. It turns out that the loss of stability of a fixed point in the frozen system due to a Neimark-Sacker bifurcation, explains the dichotomous behavior of the virtual fly.
\end{abstract}

Keywords: Discrete time dynamical systems, bifurcation analysis, pursuit behavior of blowflies.

\section{Introduction}

Male blowflies chase and catch female flies in the context of mating behavior. The authors of [3] perform the following biological experiment in order to understand the underlying system, controlling the artistic movements of male flies during high speed pursuits.

First the female fly is replaced by a black dummy target and the trajectory is simplified to a circular track on which the target flies with constant speed. Then it was observed whether male flies succeed in catching the target. As it turns out, the success of catching depends essentially on the size and on the speed of the target.

*Supported by Forschungsschwerpunkt Mathematisierung (FSPM) 'Dynamik und Struktur komplexer Systeme' 
Surprisingly, male flies do not catch big targets; instead they follow them in a constant distance, while small objects are caught easily.

Böddeker and Egelhaaf conjecture that there is no extra circuit inside the brain of the fly needed, to explain this dichotomous behavior. To test this hypothesis, the model of a virtual fly is introduced in [2] and extended in [1]. Numerical simulations for this model confirm this conjecture. The virtual fly shows the same characteristics as real flies, without having an instance that decides whether the fly catches the target or follows it in a constant distance.

In this paper, we formulate the model of the virtual fly using the notation of a discrete time dynamical system. We perform a bifurcation analysis to understand the change of behavior from a mathematical point of view.

In Section 3, we consider a target, flying on a linear trajectory. For the analysis, it is convenient to "freeze" the system, such that the position of the target is fixed. A fixed point in the frozen system corresponds to a trajectory on which the fly follows the target in a constant distance. Therefore we analyze the stability of these fixed points to understand the described dichotomous behavior.

In Section 4 a similar approach is applied for targets, flying on circular trajectories.

\section{Virtual fly as dynamical system}

First, we write the model of the virtual fly in mathematical terms. It is our aim to explain the dichotomous behavior of flies that follow big targets on rigid trajectories but catch small targets on virtuous paths. For the analysis, we consider this model as a discrete time dynamical system of the form

$$
Y_{n+1}=F\left(Y_{n}\right), \quad n \in \mathbb{N}
$$

where $Y_{n}$ is the state at time $n$. The vector $Y=(s, a, \sigma, v, x, z) \in \mathbb{R}^{9}$ contains six state variables, introduced in Table 1 . In every time step, the actual state is transformed by the map $F$ into the next one. For the length of one time step we choose the minimal processing time of neuronal activities which is $\delta_{t}=1 \mathrm{~ms}$. Velocities are measured in $[\mathrm{m} / \mathrm{s}]$ and positions in $[\mathrm{mm}]$.

\begin{tabular}{|ll|}
\hline$s \in \mathbb{R}$ & internal guess of the speed of the fly \\
$a \in \mathbb{R}$ & internal guess of the error angle \\
$\sigma \in \mathbb{R}$ & angle of body orientation of the fly w.r.t. the $(1,0)$-axis \\
$v \in \mathbb{R}^{2}$ & course and speed of the fly \\
$x \in \mathbb{R}^{2}$ & position of the fly in global coordinates \\
$z \in \mathbb{R}^{2}$ & position of the target in global coordinates \\
\hline
\end{tabular}

Table 1: Variables in the model of the virtual fly. 
The map $F$ also depends on several parameters, outlined in Table 2.

\begin{tabular}{|c|c|c|c|}
\hline parameter & value & dim. & interpretation \\
\hline$T$ & $3-15$ & $\mathrm{~mm}$ & size of the target \\
\hline$S_{m}$ & 0.8 & $\mathrm{~m} / \mathrm{s}$ & minimal speed of the fly \\
\hline$G$ & 0.125 & 1 & describes the gain of the orientation change \\
\hline$T_{s}$ & 80 & 1 & $\begin{array}{l}\text { parameter for a low-pass filter, realizing a } \\
\text { neuronal reaction time of } 80 \mathrm{~ms} \text { for the speed } \\
\text { control }\end{array}$ \\
\hline$T_{a}$ & 15 & 1 & $\begin{array}{l}\text { parameter for a low-pass filter, realizing a } \\
\text { neuronal reaction time of } 15 \mathrm{~ms} \text { for the target } \\
\text { fixation }\end{array}$ \\
\hline$q$ & 0.0865 & 1 & parameter for speed control \\
\hline$S_{v}$ & 67 & $\mathrm{~m} / \mathrm{s}$ & parameter for speed control \\
\hline$M$ & 0.0455 & 1 & kinematic constant \\
\hline
\end{tabular}

Table 2: Parameters and their typical settings in the model of the virtual fly.

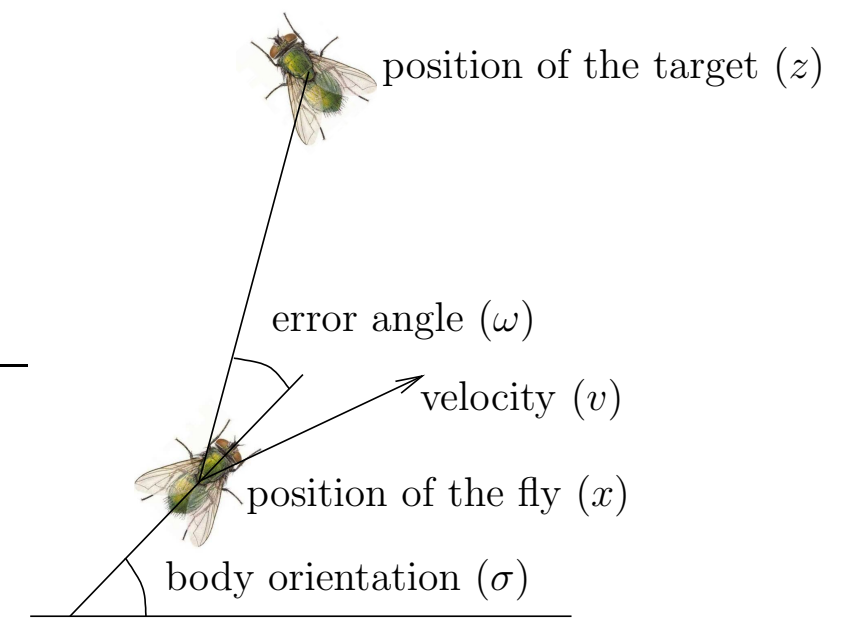

Figure 1: Settings in the model of the virtual fly.

First, two functions used to locate the target are introduced. The angular (or retinal) size $\rho=\rho(x, z, T)$ of the target is given by

$$
\rho(x, z, t)=2 \arccos \left(\frac{\|x-z\|_{2}}{\sqrt{\|x-z\|_{2}^{2}+T^{2} / 4}}\right) .
$$


Let $\alpha(x)$ be the angle, between the vector $x$ and the $(1,0)$ axis. Then

$$
\omega=\alpha(z-x)-\sigma
$$

defines the error angle $\omega$ (see Figure 1).

The speed of the virtual fly is controlled by the retinal size of the target. In case the retinal size is small the speed should increase, whereas the fly has to slow down when the retinal size of the target is big. Since the fly cannot measure all input data precisely, the following nonlinear characteristic curve is used to calculate a guess of the speed in the $n+1$-th time step:

$$
\tilde{s}_{n+1}=\rho\left(x_{n}, z_{n}, T\right) S_{v} e^{\frac{-\rho\left(x_{n}, z_{n}, T\right)}{q}}+S_{m} .
$$

Here $S_{m}$ is the minimal speed of the fly and $S_{v}$ and $q$ are parameters used to fit the model to the biological data. In the next step we use a first order low-pass filter (see Appendix A) to take neuronal reaction time into account and get

$$
s_{n+1}=\frac{\tilde{s}_{n+1}+T_{s} s_{n}}{1+T_{s}}=\frac{\rho\left(x_{n}, z_{n}, T\right) S_{v} e^{\frac{-\rho\left(x_{n}, z_{n}, T\right)}{q}}+S_{m}+T_{s} s_{n}}{1+T_{s}} .
$$

Since the reaction time for movement control is $80 \mathrm{~ms}$, we choose $T_{s}=80$.

To compute the heading of the virtual fly, the error angle $\omega$ (see Figure 1) is superposed with some uncertainty to get realistic results. With some constant $G$ that we fit to biological data, we get

$$
\tilde{a}_{n+1}=G \sin \left(\omega_{n}\right)=G \sin \left(\alpha\left(z_{n}-x_{n}\right)-\sigma_{n}\right),
$$

where $\sigma_{n}$ is the body orientation of the fly at time $n$. Applying a low-pass filter to these data ( $T_{a}=15$ is the neuronal time for object fixation) leads to

$$
a_{n+1}=\frac{\tilde{a}_{n+1}+T_{a} a_{n}}{1+T_{a}}=\frac{G \sin \left(\alpha\left(z_{n}-x_{n}\right)-\sigma_{n}\right)+T_{a} a_{n}}{1+T_{a}} .
$$

The body orientation of the fly changes in every time step and can simply be modeled by

$$
\sigma_{n+1}=\sigma_{n}+a_{n}
$$

Due to kinematic restrictions, the virtual fly cannot correct the trajectory directly towards the target. The new velocity vector $v$, containing speed and flight direction, is determined by:

$$
v_{n+1}=(1-M) v_{n}+M s_{n}\left(\begin{array}{c}
\cos \left(\sigma_{n}\right) \\
\sin \left(\sigma_{n}\right)
\end{array}\right)
$$

Since one time step is $1 \mathrm{~ms}$, kinematic restrictions on this time scale are quite strong $(M=0.0455)$. 
Then the new position of the fly is

$$
x_{n+1}=x_{n}+\delta_{t} v_{n}
$$

Recall that $\delta_{t}=1 \mathrm{~ms}$ is the length of one time step.

Finally, the position of the target is defined by

$$
z_{n+1}=f\left(z_{n}\right)
$$

where $f: \mathbb{R}^{2} \rightarrow \mathbb{R}^{2}$ is a continuous map.

We summarize the construction by writing our system in a compact form. For $Y=(s, a, \sigma, v, x, z)$ we define a smooth map $F: \mathbb{R}^{9} \rightarrow \mathbb{R}^{9}$ by

$$
F(Y)=F\left(\begin{array}{c}
s \\
a \\
\sigma \\
v \\
x \\
z
\end{array}\right)=\left(\begin{array}{c}
\frac{1}{1+T_{s}}\left(\rho(x, z, T) S_{v} e^{\frac{-\rho(x, z, T)}{q}}+S_{m}+T_{s} s\right) \\
\frac{1}{1+T_{a}}\left(G \sin (\alpha(z-x)-\sigma)+T_{a} a\right) \\
\sigma+a \\
(1-M) v+M s\left(\begin{array}{c}
\cos (\sigma) \\
\sin (\sigma)
\end{array}\right) \\
x+v \\
f(z)
\end{array}\right)
$$

Then the behavior of the virtual fly is determined by (1).

Note that capturing is not implemented into the model, thus the virtual fly can pass through the target. One the one hand, this makes no sense from a biological point of view. But as we will see, the long time behavior of this model reflects the probability of catching the target. On the other hand one can stop the simulation in case

$$
\left\|x_{n}-z_{n}\right\|_{2} \leq \text { catching distance. }
$$

\section{Targets on linear trajectories}

In this section, we consider the chasing of targets, flying on linear trajectories with constant speed: $f(z)=z+\gamma$, where $\gamma$ is a constant vector. For the analysis, we freeze this system, i.e. we shift the whole system after every time step, such that the position of the target stays constant, see Figure 2.

The correctness of this approach guarantees the following lemma. 


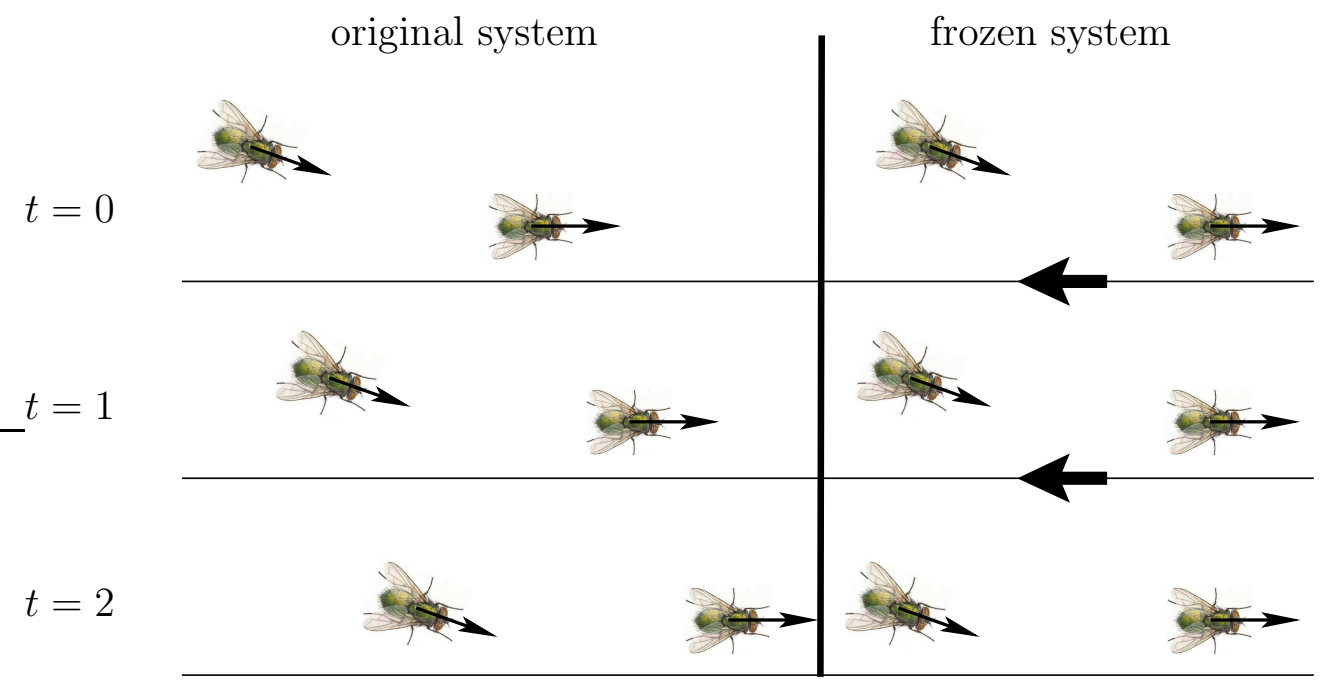

Figure 2: Original and frozen system.

Lemma 1 Let $f(z)=z+\gamma$, then

$$
F\left(\begin{array}{c}
s \\
a \\
\sigma \\
v \\
x+\gamma \\
z+\gamma
\end{array}\right)=F\left(\begin{array}{c}
s \\
a \\
\sigma \\
v \\
x \\
z
\end{array}\right)+\left(\begin{array}{l}
0 \\
0 \\
0 \\
0 \\
\gamma \\
\gamma
\end{array}\right) .
$$

Proof: Obviously, the first 4 components stay invariant w.r.t. this transformation, since $\rho$ and $\omega$ depend only on the length $\|x-z\|_{2}=\|x-\gamma-z+\gamma\|_{2}$, see (2), (3). Furthermore, the $x$ and $z$ component are linear, thus (5) holds.

Therefore, we consider the "frozen" map $\tilde{F}$, defined by

$$
\tilde{F}\left(\begin{array}{c}
s \\
a \\
\sigma \\
v \\
x \\
z
\end{array}\right):=F\left(\begin{array}{c}
s \\
a \\
\sigma \\
v \\
x \\
z
\end{array}\right)-\left(\begin{array}{l}
0 \\
0 \\
0 \\
0 \\
\gamma \\
\gamma
\end{array}\right)
$$

for the forthcoming analysis. Note that a fixed point $\xi$ of $\tilde{F}$ leads in the original system to a trajectory on which the fly follows the target with constant distance and speed, see Figure 3. We call these trajectories rigid. Formally, let 
$Y_{0}=(\bar{s}, \bar{a}, \bar{\sigma}, \bar{v}, \bar{x}, \bar{z})$ be a fixed point of $\tilde{F}$. Using Lemma 1 we get by induction

$$
Y_{1}=F\left(Y_{0}\right)=\left(\begin{array}{c}
\bar{s} \\
\bar{a} \\
\bar{\sigma} \\
\bar{v} \\
\bar{x} \\
\bar{z}
\end{array}\right)+\left(\begin{array}{c}
0 \\
0 \\
0 \\
0 \\
\gamma \\
\gamma
\end{array}\right)=\left(\begin{array}{c}
\bar{s} \\
\bar{a} \\
\bar{\sigma} \\
\bar{v} \\
\bar{x}+\gamma \\
\bar{z}+\gamma
\end{array}\right) \Longrightarrow Y_{n}=\left(\begin{array}{c}
\bar{s} \\
\bar{a} \\
\bar{\sigma} \\
\bar{v} \\
\bar{x}+n \gamma \\
\bar{z}+n \gamma
\end{array}\right), n \in \mathbb{N}
$$

This situation arises in our model, for example, if we choose $\gamma=(\ell, 0), \ell=1.5$, and $T=14 \mathrm{~mm}$. Then the target flies with a constant speed of $1.5 \mathrm{~m} / \mathrm{s}$ in the direction of the $x_{1}$-axis; the other parameters are chosen according to Table 2 . A simulation for this setup is illustrated in Figure 3. Here the fixed point $\xi$ of $\tilde{F}$ is
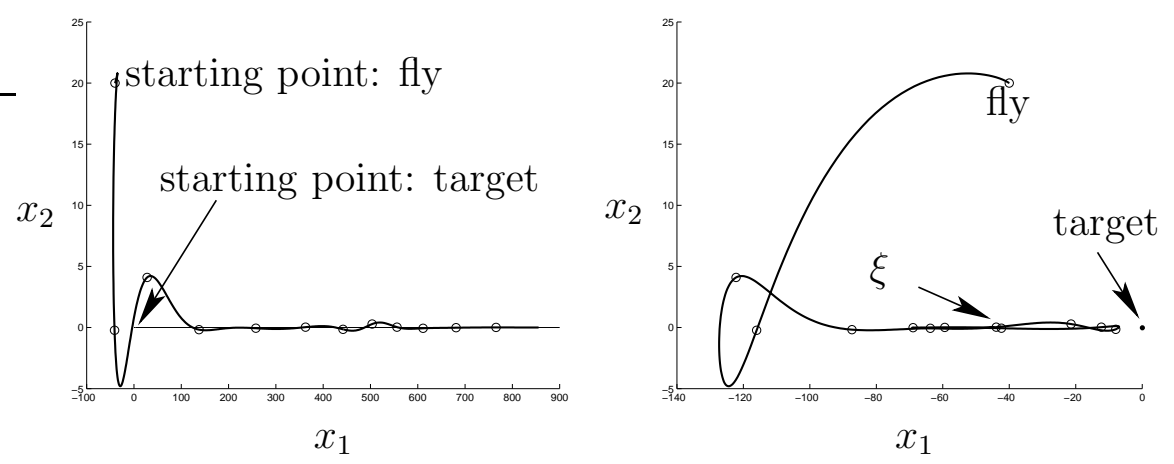

Figure 3: Original (left) and frozen system (right) for $T=14, \ell=1.5$. 600 time steps of the simulation are displayed and every 50 time steps the position of the fly is marked with a circle. For this parameter setup, the fixed point $\xi$ of the frozen system is stable.

stable (i.e. all multipliers of the matrix $\tilde{F}^{\prime}(\xi)$ lie inside the unit circle). The virtual fly is attracted by this fixed point and will follow the target forever. Note that due to the choice of $\gamma=(1.5,0)$, the target moves in the original system from $(0,0)$ to $(900,0)$ in 600 simulation steps, but in the frozen system, the position stays constant. Therefore the $x_{1}$-axes in the left and right picture of Figure 3 (and Figure 4 ) have different scales.

When one parameter varies, the fixed point can lose its stability, for example, via a fold or Neimark-Sacker bifurcation (see [4], [7]). The size of the target is one suitable bifurcation parameter. As it turns out, a Neimark-Sacker bifurcation occurs when the parameter $T$ decreases. Beyond this bifurcation the fixed point is unstable but additionally, a stable invariant curve exists.

For a target size of $6.5 \mathrm{~mm}$ a stable invariant curve is displayed in Figure 4. On trajectories, converging toward the invariant curve, the fly will catch the target, therefore instability of the rigid trajectory is a desirable feature. 

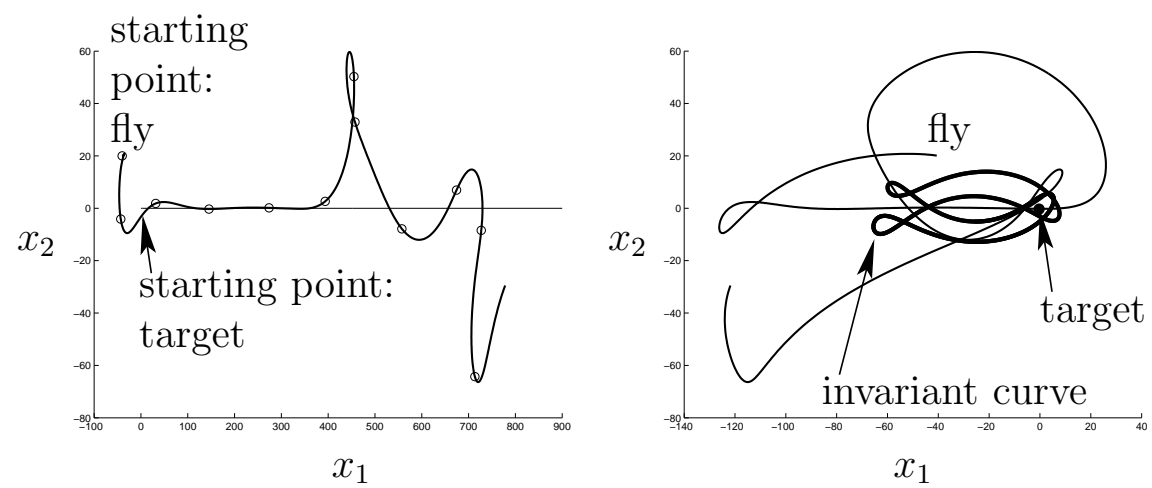

Figure 4: Original (left) and frozen system (right) for $T=6.5, \ell=1.5$. 600 time steps of the simulation are displayed and every 50 time steps the position of the fly is marked with a circle. At this parameter setup, the fixed point $\xi$ is unstable, but a stable invariant curve that comes close to the target exists. Note that it takes more than 600 time steps, until the fly approaches the invariant curve.

The success of catching also depends essentially on the speed $(\ell)$ of the target. It is in addition to the size $(T)$ a second bifurcation parameter of our system. We search for parameter constellations of $T$ and $\ell$, where a stable fixed point of the frozen system exists (all other parameters are chosen according to Table 2). Consequently, we find at these parameter setups a rigid trajectory for the original system, on which the fly follows the target forever.

We perform the computations along the following steps, using the softwarepackage Content [5]. First we fix $T=14, \ell=1.5$ and compute a fixed point of $\tilde{F}$ which is stable (Figure 3). Then we continue the fixed point w.r.t. the parameter $T$ and obtain a branch of fixed points $\xi(T)$. We monitor the eigenvalues of $\left.\tilde{F}^{\prime}(\xi(T))\right)$ on this branch. Indeed a complex conjugated pair of eigenvalues leave the unit circle at $\tilde{T} \approx 8.49$. At this parameter the fixed point loses its stability via a NeimarkSacker bifurcation and for $T<\tilde{T}$ it is unstable, see Figure 5 (i.e. rigid trajectories do not exist for the original system). 


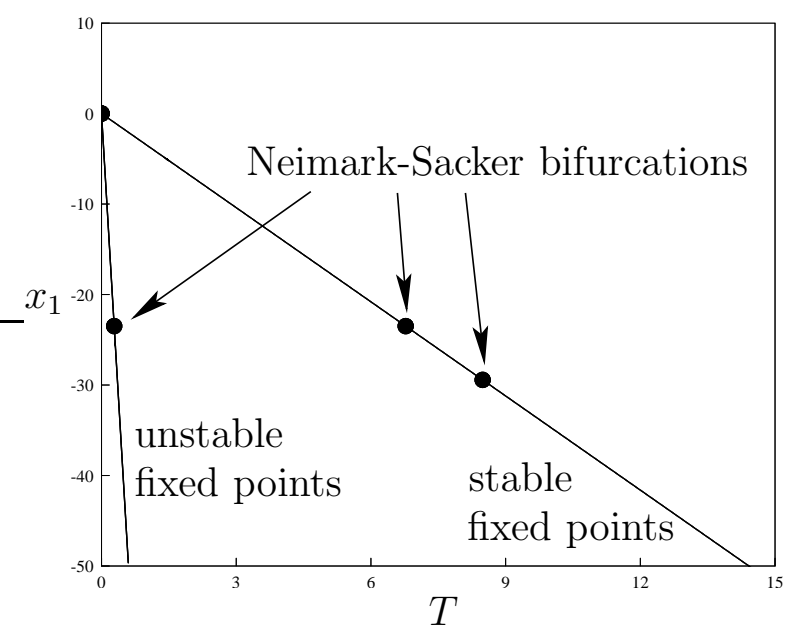

Figure 5: Continuation of fixed points for $\ell=1.5$ w.r.t. the parameter $T$. At $\tilde{T} \approx 8.40$ and $\hat{T} \approx 6.77$ the fixed point undergoes a Neimark-Sacker bifurcation. There is also a second branch of unstable fixed points.

Furthermore, at $\hat{T} \approx 6.77$ the fixed point undergoes a second Neimark-Sacker bifurcation, such that it has four unstable eigenvalues for $T<\hat{T}$. There is also a second branch of fixed points, undergoing a Neimark-Sacker bifurcation. But this branch is of less interest, since the fixed points are unstable.

In the next step, we continue the two Neimark-Sacker bifurcations at the parameter $T=\tilde{T}$ and $T=\hat{T}, \ell=1.5$, see Figure 5 w.r.t. the parameters $T$ and $\ell$ to find an area in the $(T, \ell)$-parameter plane, where stable fixed points exist, see Figure 6 .

We discuss the results of this analysis for the original system. At a parameter constellation inside the area $\mathbf{0}$, a rigid trajectory exists on which the fly follows the target forever. When parameters vary such that we cross the border to the area (2, the rigid trajectory loses its stability via a Neimark-Sacker bifurcation. Note that an unstable trajectory still exists, but since the fly can only follow stable trajectories, it is not visible in simulations. In the frozen system, a stable invariant curve emanates at the bifurcation point, cf. Figure 4. On a trajectory that converges towards this invariant curve, the fly necessarily approaches the target.

For a parameter setup inside the area 3, the fixed point has four unstable eigenvalues, since it undergoes a second Neimark-Sacker bifurcation. The stable invariant curve, emanating at the first bifurcation is not affected by the second bifurcation and therefore this bifurcation does not influence the long-time behavior of the fly essentially. 


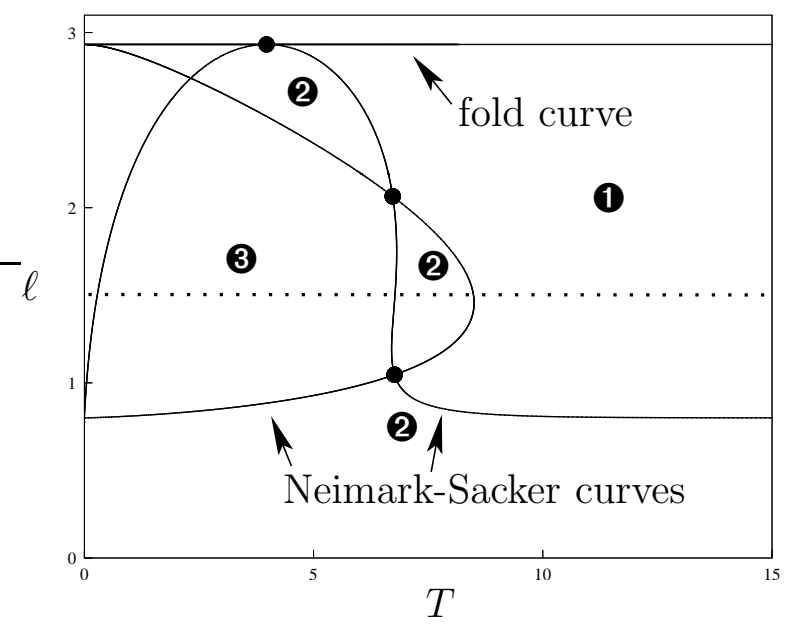

Figure 6: Continuation of the two Neimark-Sacker bifurcations, displayed in Figure 5, w.r.t. the parameters $T$ and $\ell . A t \bar{\ell} \approx 2.932$ the fixed point undergoes a fold bifurcation (independent of $T$ ). Thus for $\ell>\bar{\ell}$ a fixed point cannot exist. In the area $\mathbf{1}$ a stable fixed point exists, which becomes unstable when moving into the region 2 and $\mathbf{3}$. The cross-section, analyzed in Figure 5 is indicated by a dotted line.

\section{Targets on circular trajectories}

The authors of [3] investigate in their biological experiments the chasing of targets, flying on circular trajectories with constant speed. For a bifurcation analysis, we freeze the system by applying in every time step a rotation, such that the position of the target is fixed (cf. Section 3). In Figure 7 this idea is illustrated for a fly, following the target on a rigid circular trajectory. Thus in this situation, the position of both insects is fixed in the frozen system.

The following lemma shows the validity of this idea.

Lemma 2 Let $f(z)=D_{\varphi}(z)$, with the rotation matrix $D_{\varphi}=\left(\begin{array}{cc}\cos \varphi & -\sin \varphi \\ \sin \varphi & \cos \varphi\end{array}\right)$. Denote by $F_{s}, F_{a}, F_{\sigma}, F_{v}, F_{x}$ and $F_{z}$ the components of $F$ and let $Y=(s, a, \sigma, v, x, z)$. Then

$$
F\left(\begin{array}{c}
s \\
a \\
\sigma+\varphi \\
D_{\varphi} v \\
D_{\varphi} x \\
D_{\varphi} z
\end{array}\right)=\left(\begin{array}{c}
F_{s}(Y) \\
F_{a}(Y) \\
F_{\sigma}(Y)+\varphi \\
D_{\varphi} F_{v}(Y) \\
D_{\varphi} F_{x}(Y) \\
D_{\varphi} F_{z}(Y)
\end{array}\right) .
$$



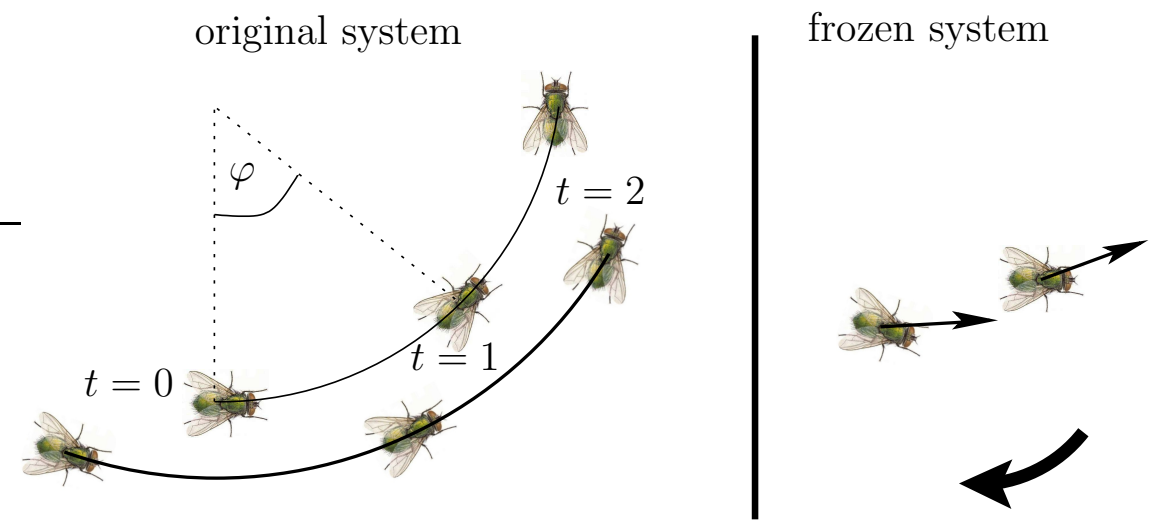

Figure 7: Original and frozen system in case of a fly following the target on a rigid circular trajectory.

Proof: Since

$$
\left\|D_{\varphi} x-D_{\varphi} z\right\|_{2}=\left\|D_{\varphi}(x-z)\right\|_{2}=\|x-z\|_{2}
$$

and

$$
\alpha\left(D_{\varphi} z-D_{\varphi} x\right)-(\sigma+\varphi)=\alpha\left(D_{\varphi}(z-x)\right)-\sigma-\varphi=\alpha(z-x)-\sigma
$$

hold, we get

$$
\begin{aligned}
& F_{s}\left(s, a, \sigma+\varphi, D_{\varphi} v, D_{\varphi} x, D_{\varphi} z\right)=F_{s}(Y), \\
& F_{a}\left(s, a, \sigma+\varphi, D_{\varphi} v, D_{\varphi} x, D_{\varphi} z\right)=F_{a}(Y) .
\end{aligned}
$$

Furthermore,

$$
\begin{aligned}
F_{\sigma}\left(s, a, \sigma+\varphi, D_{\varphi} v,\right. & \left.D_{\varphi} x, D_{\varphi} z\right)=\sigma+\varphi+a=\varphi+F_{\sigma}(Y), \\
F_{v}\left(s, a, \sigma+\varphi, D_{\varphi} v, D_{\varphi} x, D_{\varphi} z\right) & =(1-M) D_{\varphi} v+M s\left(\begin{array}{c}
\cos (\sigma+\varphi) \\
\sin (\sigma+\varphi)
\end{array}\right) \\
= & (1-M) D_{\varphi} v+M s\left(\begin{array}{c}
\cos \varphi \cos \sigma-\sin \varphi \sin \sigma \\
\sin \varphi \cos \sigma+\cos \varphi \sin \sigma
\end{array}\right) \\
= & (1-M) D_{\varphi} v+M s\left(\begin{array}{cc}
\cos \varphi-\sin \varphi \\
\sin \varphi & \cos \varphi
\end{array}\right)\left(\begin{array}{c}
\cos \sigma \\
\sin \sigma
\end{array}\right) \\
= & D_{\varphi}\left((1-M) v+M s\left(\begin{array}{c}
\cos \sigma \\
\sin \sigma
\end{array}\right)\right) \\
= & D_{\varphi} F_{v}(Y), \\
F_{x}\left(s, a, \sigma+\varphi, D_{\varphi} v, D_{\varphi} x, D_{\varphi} z\right) & =D_{\varphi} x+D_{\varphi} v=D_{\varphi}(x+v)=D_{\varphi} F_{x}(Y), \\
F_{z}\left(s, a, \sigma+\varphi, D_{\varphi} v, D_{\varphi} x, D_{\varphi} z\right) & =D_{\varphi}\left(D_{\varphi} z\right)=D_{\varphi} F_{z}(Y) .
\end{aligned}
$$


The "frozen" system $\hat{F}$ is defined by

$$
\hat{F}(Y):=F\left(\begin{array}{c}
s \\
a \\
\sigma-\varphi \\
D_{-\varphi} v \\
D_{-\varphi} x \\
D_{-\varphi} z
\end{array}\right)=\left(\begin{array}{c}
F_{s}(Y) \\
F_{a}(Y) \\
F_{\sigma}(Y)-\varphi \\
D_{-\varphi} F_{v}(Y) \\
D_{-\varphi} F_{x}(Y) \\
D_{-\varphi} F_{z}(Y)
\end{array}\right)
$$

Let $Y_{0}=(\bar{s}, \bar{a}, \bar{\sigma}, \bar{v}, \bar{x}, \bar{z})$ be a fixed point of $\hat{F}$. Then we get, using Lemma 2 inductively

$$
Y_{1}=F\left(Y_{0}\right)=\left(\begin{array}{c}
\bar{s} \\
\bar{a} \\
\bar{\sigma}+\varphi \\
D_{\varphi} \bar{v} \\
D_{\varphi} \bar{x} \\
D_{\varphi} \bar{z}
\end{array}\right) \quad \Longrightarrow \quad Y_{n}=\left(\begin{array}{c}
\bar{s} \\
\bar{a} \\
\bar{\sigma}+n \varphi \\
D_{n \varphi} \bar{v} \\
D_{n \varphi} \bar{x} \\
D_{n \varphi} \bar{z}
\end{array}\right), n \in \mathbb{N} .
$$

This gives a rigid trajectory for the original system.

Parallel to the analysis in Section 3 we simulate a trajectory of the fly for the original and for the frozen system in case the rigid trajectory is stable (Figure 8) and in case it is unstable (Figure 9$)$. We choose $z=(100,0)$ as starting point of the target and a rotation angle $\varphi=0.0125$ (radius $0.1 \mathrm{~m}$ ). In one second the target flies $\frac{1000 \varphi}{2 \pi}$ loops (note that one time step is $1 \mathrm{~ms}$ ). Since the circumference is $\frac{2 \pi}{10} \mathrm{~m}$, the speed of the target is $\ell=100 \varphi \mathrm{m} / \mathrm{s}$.
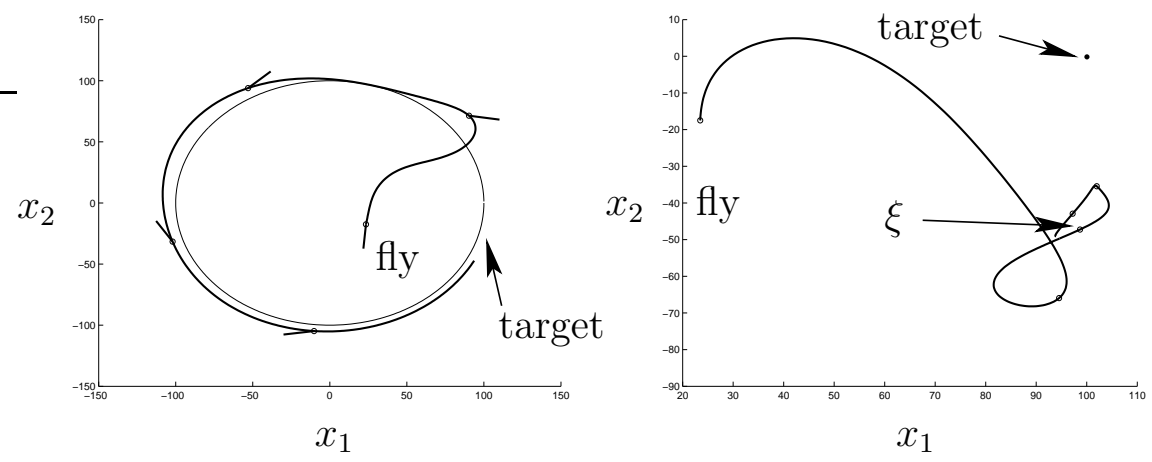

Figure 8: Original (left) and frozen system (right) for $T=14, \ell=1.25$. 500 time steps of the simulation are displayed and every 100 time steps and the position and orientation of the fly is symbolized in the left picture by "—". At this parameter setup, the fixed point $\xi$ of the frozen system is stable. 

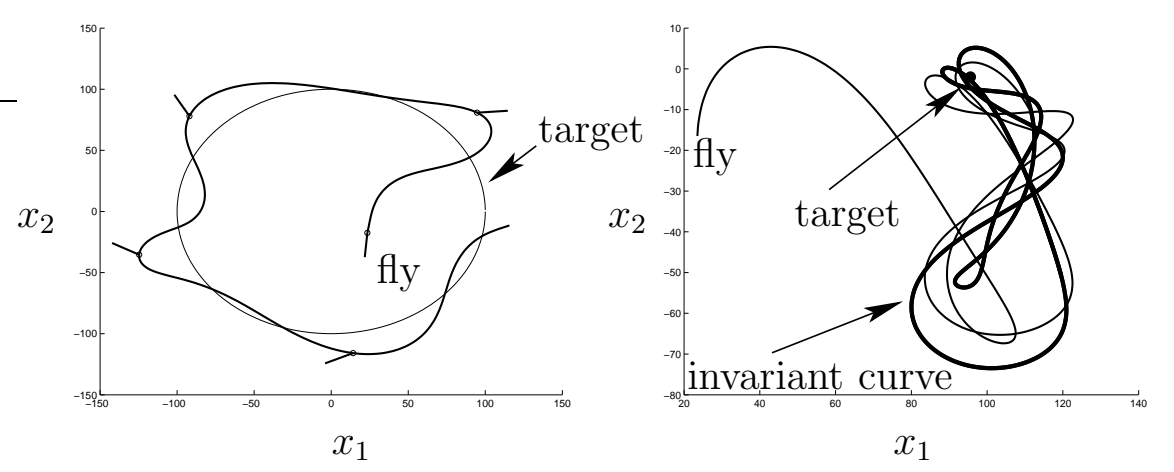

Figure 9: Original (left) and frozen system (right) for $T=7, \ell=1.25$. 500 time steps of the simulation are displayed and every 100 time steps and the position and orientation of the fly is symbolized in the left picture by " $\multimap$ ". At this parameter setup, the fixed point $\xi$ is unstable, but a stable invariant curve that comes close to the target exists.

We continue the stable fixed point w.r.t. the parameter $T$ (the size of the target) using Content [5], see Figure 10. One branch of fixed points undergoes a NeimarkSacker bifurcation, thus the behavior of this system is almost the same as for linear trajectories, analyzed in Section 3. But there is a second stable branch of fixed points, existing only for small $T$, which also gives a rigid trajectory for the original system. From a biological point of view this branch is less important since female flies (the targets) have an average size of $8 \mathrm{~mm}$. Note that on this branch the fixed point loses its stability via a fold bifurcation (cf. [4], [7]).

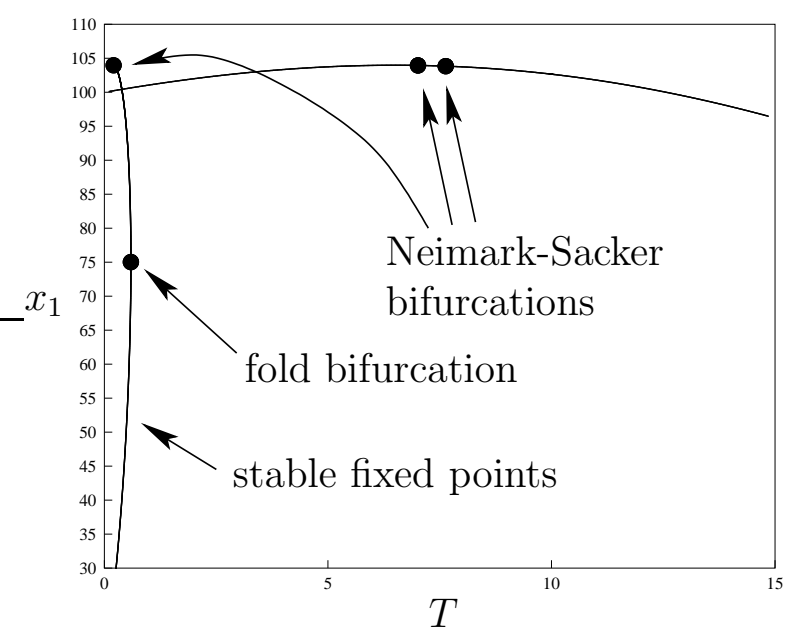

Figure 10: Continuation of fixed points for $\ell=1.25$ w.r.t. the parameter T. At $\tilde{T} \approx 8.40$ and $\hat{T} \approx 6.77$ the fixed point undergoes a NeimarkSacker bifurcation. For small $T$, a second branch of fixed points exists, undergoing a fold bifurcation. 


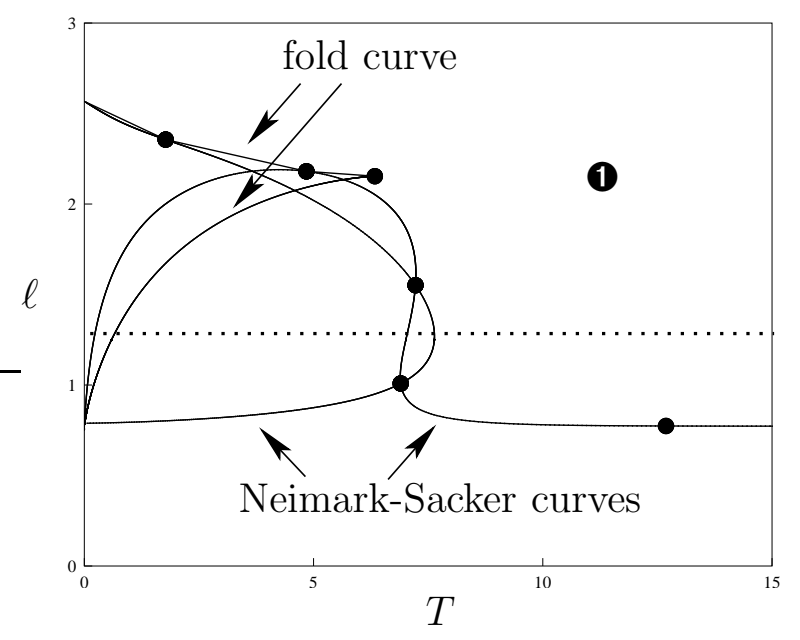

Figure 11: Continuation of the two Neimark-Sacker bifurcations, displayed in Figure 10, w.r.t. the parameters $T$ and $\ell$. In the area $\mathbf{0}$ the fixed point is stable. The cross-section, analyzed in Figure 10 is indicated by a dotted line.

Figure 11 shows the result of the continuation of the two Neimark-Sacker and of the fold bifurcation w.r.t. the parameters $T$ and $\ell$. Since we have two branches of fixed points, this bifurcation diagram seems to be more complicated, but in the area $\mathbf{0}$ a stable fixed point and thus a stable rigid trajectory exists. But when we choose a parameter outside $\mathbf{0}$ the rigid trajectory is unstable and as described in Section 3 the fly will succeed in catching the target. Again these instabilities are a welcome effect.

\section{Appendix A First order low-pass filters}

To simulate neuronal reaction times and the smoothing property of neuronal networks, so called low-pass filters are useful, cf. [6]. Consider the differential equation

$$
\tau u^{\prime}+u=w,
$$

where $w$ is the input, $u$ the output and $\tau$ the delay time. A discretization of (7) leads to the difference equation

$$
u_{n+1}=\frac{w_{n+1}+\tau u_{n}}{\tau+1} .
$$

Note that this approach does not lead to delay difference equations. 


\section{Acknowledgment}

The author wishes to thank Prof. Dr. Martin Egelhaaf and Dr. Norbert Böddeker for their cooperation and especially for an introduction into the model of the virtual fly. The author thanks Prof. Dr. Wolf-Jürgen Beyn for very helpful suggestions about this paper.

\section{References}

[1] N. Böddeker and M. Egelhaaf. Chasing behaviour of blowflies: A smooth pursuit tracking system generates saccades. Technical report, 2003.

[2] N. Böddeker and M. Egelhaaf. Steering a virtual blowfly: simulation of visual pursuit. Proc. R. Soc. Lond. B, pages 1971-1978, 2003.

[3] N. Böddeker, R. Kern, and M. Egelhaaf. Chasing a dummy target: smooth pursuit and velocity control in male blowflies. Proc. R. Soc. Lond. B, pages 393-399, 2003.

[4] Y. A. Kuznetsov. Elements of Applied Bifurcation Theory. Springer-Verlag, New York, second edition, 1998.

[5] Y. A. Kuznetsov and V. V. Levitin. Content - integrated environment for analysis of dynamical systems, 1998. http://www.cwi.nl/ftp/CONTENT.

[6] D. Varjú. Systemtheorie für Biologen und Mediziner. Springer-Verlag, Berlin, 1977.

[7] S. Wiggins. Introduction to Applied Nonlinear Dynamical Systems and Chaos. Springer-Verlag, New York, 1990. 